\title{
The Effects of CACNAIC Gene Polymorphism on Spatial Working Memory in Both Healthy Controls and Patients with Schizophrenia or Bipolar Disorder
}

\author{
Qiumei Zhang ${ }^{1,4}$, Qiuge Shen ${ }^{2,4}$, Zhansheng $X^{2,4}$, Min Chen',4, Lina Cheng ${ }^{2}$, Jinguo Zhai', Huang Gu ${ }^{2}$, \\ Xin $\mathrm{Bao}^{2}$, Xiongying Chen ${ }^{2}$, Keqin Wang', Xiaoxiang Deng ${ }^{2}$, Feng $\mathrm{Ji}^{\prime}$, Chuanxin Liu', Jun $\mathrm{Li}^{*}, 2$, Qi Dong ${ }^{2}$ \\ and Chuansheng Chen ${ }^{3}$
}

'School of Mental Health, Jining Medical University, Jining, China; ${ }^{2}$ State Key Laboratory of Cognitive Neuroscience and Learning, Beijing Normal University, Beijing, China; ${ }^{3}$ Department of Psychology and Social Behavior, University of California, Irvine, CA, USA

\begin{abstract}
CACNA IC gene polymorphism ( $r$ I 006737) is a susceptibility factor for both schizophrenia (SCZ) and bipolar disorder (BP). However, its role in working memory, a cognitive function that is impaired in both diseases, is not clear. Using three samples, including healthy controls, patients with SCZ, and patients currently in manic episodes of BP, this study tested the association between the SNP rs I006737 and spatial working memory as measured by an N-back task and a dot pattern expectancy (DPX) task. Among SCZ patients and healthy controls, the clinical risk allele was associated with impaired working memory, but the association was either in opposite direction or nonsignificant in patients with BP. These results indicated that rs 1006737 may have differential effects on working memory in different disease populations and pointed to the necessity for more studies in different patient populations.

Neuropsychopharmacology (2012) 37, 677-684; doi:10.1038/npp.201 I.242; published online 19 October 2011
\end{abstract}

Keywords: schizophrenia; CACNAIC; working memory; polymorphism

\section{INTRODUCTION}

It is widely accepted that schizophrenia (SCZ) and bipolar disorder (BP) share genetic susceptibility (Cardno et al, 2002). Some genes have been suggested to show significant associations with both diseases (Moskvina et al, 2009). One such gene is CACNA1C, which encodes the alpha-1C subunit of the L-type voltage-gated calcium channel $\left(\mathrm{Ca}_{\mathrm{v}} 1.2\right)$ (Green et al, 2010). Calcium channels are important for converting electrical activity into biochemical events. Through these voltage-dependent channels, calcium enters the cell and regulates multiple enzymes, ion pumps, and components of cytoskeleton (Obermair et al, 2008; Catterall, 2000). Therefore, variations in calcium channels can affect signal transduction and brain circuits, which may in turn contribute to the etiology of both BP and SCZ. Two large genome-wide association studies of $\mathrm{BP}$ involving three independent samples found that the strongest and most

*Correspondence: Dr J Li, State Key Laboratory of Cognitive Neuroscience and Learning, Beijing Normal University, 19\# Xinjiekouwai Road, Beijing 100875, China, Tel: + 86105880 1755, Fax: + 8610 5880 1755, E-mail: lijundp@bnu.edu.cn

${ }^{4}$ These authors contributed equally to this work.

Received 27 April 2011; revised 6 September 2011; accepted 12 September 2011 consistent results were at an intronic polymorphism of this gene, namely rs1006737 (Ferreira et al, 2008; Sklar et al, 2008). In addition, the same polymorphism was also suggested to confer risk for SCZ (Green et al, 2010; Nyegaard et al, 2010). However, the exact function of this polymorphism is unknown.

In addition to having common susceptibility genes, BP and SCZ also share intermediate phenotypes, which include prefrontal dysfunction and its related cognitive impairments such as poor working memory (Hill et al, 2008, also see Manoach, 2003, for an earlier review). Recent studies have suggested a potential link between CACNA1C rs1006737 and these intermediate phenotypes. First, a study of post-mortem brain samples found increased expression of CACNA1C mRNA in human dorsolateral prefrontal cortex (DLPFC) for the BP/SCZ risk allele (Bigos et al, 2010), indicating a possible functional significance of this SNP at this region. Second, using different tasks (a spatial $\mathrm{N}$-back task and a verbal fluency task), two independent fMRI studies of healthy volunteers consistently found that those with the risk allele showed increased prefrontal activity when performing the working memory tasks (Bigos et al, 2010; Krug et al, 2010). However, no studies thus far have examined the CACNA1C gene and working memory among patients with either BP or SCZ. 
The current study explored associations between the SNP rs 1006737 and working memory in three samples including healthy volunteers, SCZ patients, and BP patients. As suggested by the Cognitive Neuroscience Treatment Research to Improve Cognition in Schizophrenia (CNTRICS) project, working memory that is severely impaired in SCZ patients includes at least two important subunits, namely interference control and goal maintenance (Barch et al, 2009). The spatial N-back task mainly assesses interference control, and poor performance on this task has been viewed as an intermediate phenotype of SCZ (Blokland et al, 2008; Bigos et al, 2010). Goal maintenance has been assessed by either the original AX-CPT task or its revised version - the dot pattern expectancy (DPX) task, which used spatial dot patterns as stimuli (Barch et al, 2009). Previous studies found that both SCZ patients and their healthy relatives or siblings showed impaired performance at the DPX task (MacDonald et al, 2005; MacDonald et al, 2003), indicating that this task may also be a possible intermediate phenotype. The current study used both the N-back and the DPX tasks and hypothesized that the clinical risk allele of rs1006737 would be associated with poorer working memory.

\section{SUBJECTS AND METHODS}

\section{Subjects}

The sample consisted of 318 patients with SCZ, 74 patients with BP, and 401 healthy controls. All subjects were Han Chinese. The patients were recruited from the inpatients of the Ankang Hospital in Shangdong province, a division of the Jining Medical College, from August 2008 to January 2011. All patients had been hospitalized for $<1$ month and fulfilled the ICD-10 criteria for SCZ or BP based on the diagnostic consensus of two experienced psychiatrists using the Mini International Neuropsychiatric Interview (MINI). This scale has a Chinese version with high reliability and validity (Si et al, 2009). Subjects were excluded if one of the psychiatrists was uncertain about a given patient's diagnosis. The general recruitment procedure was that a clinician first judged if the patient satisfied the inclusion and the exclusion criteria (see below). The clinician and the psychologist then together explained the study to the patient, including drawing of blood and cognitive tests, and answered all questions. Subjects then signed informed consent document. Afterwards, the blood was drawn and the psychologist asked the patient to perform the cognitive tasks.

The positive and negative syndrome scale (PANSS) was used to assess each SCZ patient's positive (SAPS) and negative (SANS) symptoms at the time of the administration of the cognitive tests. The mean score of the patients' SAPS was $19.31 \pm 7.09$ and the mean SANS score was $18.01 \pm 7.27$. The mean duration of illness was $6.16 \pm 8.01$ years, and the mean number of previous hospitalizations was $1.39 \pm 2.23$. All patients were undergoing monotherapy with atypical antipsychotics and had been treated for $>2$ weeks. Exclusion criteria for the patients included a history of other psychiatric disorders, a history of severe head injury (including any closed or open head injuries that may be related to current symptoms or impact cognitive functions), currently having acute psychotic episodes, current substance abuse, and failure to cooperate during the cognitive tests. Subjects were deemed by the experimenter as 'fail to cooperate' when they abruptly stopped performing tasks in the middle of the experiment, when they pressed keys only when prompted by the experimenter, and when they failed to cooperate to complete the practice trials of a test to reach an acceptable threshold of accuracy after multiple attempts. All patients with BP were currently in episodes of mania. Symptoms were assessed by Young Mania Rating Scale (YMRS) at the time of the administration of the cognitive tests. The mean score was $15.86 \pm$ 12.80. All patients were under treatment with atypical antipsychotics and had been treated for $>2$ weeks. Exclusion criteria for the patients included a history of other psychiatric disorders, any severe head injury, current substance abuse, and failure to cooperate during the cognitive tests.

The healthy controls were from the same geographical region as the patients and were interviewed by experienced psychiatrists to screen for any personal or family history of psychiatric disorders. Additional demographic information for both patients and the healthy controls is shown in Table 1. This study was approved by the Institutional Review Board of the Institute of Cognitive Neuroscience and Learning at Beijing Normal University, and all subjects gave written informed consent for this study.

Table I Differences in the Demographic Data Among Patients with SCZ, Patients with BP, and Healthy Controls

\begin{tabular}{|c|c|c|c|c|c|c|}
\hline & SCZ & Controls & BP & SCZ vs Controls & BP vs Controls & $S C Z$ vs $B P$ \\
\hline & & & & F or $\chi^{2}(P$-value $)$ & F or $\chi^{2}(P$-value $)$ & $\mathbf{F}$ or $\chi^{2}$ (P-value $)$ \\
\hline Age (years) & $27.94 \pm 7.89$ & $22.43 \pm 6.30$ & $27.08 \pm 8.28$ & $105.33(<0.00 \mid)^{*}$ & $29.66(<0.00 \text { I })^{*}$ & $0.66(0.416)$ \\
\hline Male/female & $213 / 105$ & $250 / 151$ & $54 / 20$ & $2.52(0.283)$ & $3.06(0.080)$ & $0.992(0.319)$ \\
\hline Education (years) & $|0.29 \pm 2.9|$ & $10.60 \pm 2.78$ & $10.04 \pm 2.69$ & $2.99(0.084)$ & $2.97(0.085)$ & $0.4 \mid(0.522)$ \\
\hline
\end{tabular}

Neuropsychopharmacology 


\section{Cognitive Tasks}

All subjects completed the N-back task and the Wechsler Adult Intelligence Scale-Revised (WAIS-RC). The DPX task was completed by almost all healthy controls (396 of 401), but by only a subsample of the patients (104 patients with SCZ and 27 patients with BP) because this task was added late in the long and continual process of recruiting patients.

The N-back task was similar to the version introduced by Callicott et al (1998). In this task, a white circle was presented randomly at one of the four corners of a gray diamond-shaped square in a white background on an IBM 14-inch screen notebook. The four response buttons were arranged also in a diamond shape similar to the configuration of the white circles presented on the screen. Subjects used their right index or middle finger to press one of the four buttons to match the target stimulus. There were three task conditions: 0-, 1-, and 2-back. In the 0-back task, the subjects were instructed to press the button whose position was the same as the white circle on the screen at the current trial. In the 1-back task, the subjects pressed the button corresponding to the position of the white circle presented one trial before the current one. In the 2-back task, the subjects pressed the button corresponding to the position of the white circle presented two trials before the current one. Each condition (performed in one block) included 48 trials. All subjects followed the order of 0-, 1-, and 2-back conditions. The stimulus presentation time was $200 \mathrm{~ms}$ and the inter-stimulus interval was $800 \mathrm{~ms}$. Following Blokland et al's twin study (2008), the current study used error rate (percentage of wrong responses) to index performance.

The DPX task was similar to that introduced by MacDonald et al (2005). The task was presented in 4 blocks of 40 stimulus pairs (the cues and the probes) that were presented sequentially in the center of a 14-inch screen. The stimuli were Braille font dot patterns and were presented in pairs (cue-probe). There were four conditions: AX, AY, BX, and $\mathrm{BY}$, where $\mathrm{A}$ and $\mathrm{B}$ designate different cues and $\mathrm{X}$ and $\mathrm{Y}$ different probes. After $350 \mathrm{~ms}$ of a fixation cross, a cue was presented for $1000 \mathrm{~ms}$, followed by a $4000-\mathrm{ms}$ delay, and then the probe was presented for $500 \mathrm{~ms}$. Between trials was a $650-\mathrm{ms}$ white intermission screen. Subjects were instructed to press a target key in the AX condition but to press the non-target key in all other conditions (see MacDonald et al, 2005, for details). As in MacDonald et al (2005) study, the 40 trials of each block consisted of 28 (70\%) AX trials, 5 (12.5\%) AY trials, 5 (12.5\%) BX trials, and $2(5 \%) \mathrm{BY}$ trials. The error rate of the BX condition was used as the measurement of working memory because previous studies (MacDonald et al, 2003, 2005) showed that, compared with healthy controls, both SCZ patients and their healthy relatives showed significantly higher error rates in this condition, but not in the other three conditions. We also used BX-AY error difference scores, which have been suggested to have genetic components (MacDonald et al, 2003, 2005).

\section{SNPs Genotyping}

Rs1006737 was genotyped using Taqman allele-specific assays on the 7900HT Fast Real-Time PCR System (Applied Biosystems, Foster City, CA). The sample success rate for this SNP was $100 \%$ (ie, no failures across participants to 'call' the polymorphism) and the reproducibility of the genotyping was $100 \%$ according to a duplicate analysis of at least $2 \%$ of the genotypes.

\section{Analysis}

The Hardy-Weinberg test of the SNP was done by using the PLINK program (Purcell et al, 2007). All other analyses were done by using SPSS version 13.0. Non-genetic factors, including age, gender, IQ, and years of education among genotypic groups were compared using either one-way ANOVA or $\chi^{2}$ test. Due to the small number of the AA genotype (two in the control group and one in the SCZ group), we combined the AA genotype with the AG genotype. The associations between cognitive function and SNP were tested by two-way ANCOVA. The two fixed factors were genotype (minor allele (A) carriers $v s$ major allele G homozygotes) and diagnosis (SCZ vs BP vs controls). Demographic factors including age, gender, years of education, and IQ were entered as covariates. Significant interaction effects between genotype and diagnosis or significant main effect of genotype were further explored to examine the simple effects of genotype in the three samples separately. In this analysis, age, gender, education years, and IQ were also used as covariates.

\section{RESULTS}

There were significant differences in mean age between SCZ patients and controls and between BP patients and controls (both $P$-values $<0.05$, see Table 1 ). There were also significant differences in IQ among the three samples (SCZ vs control, $\mathrm{F}=86.34, P$-values $<0.001$; BP $v s$ control, $\mathrm{F}=8.70, P=0.003$; SCZ vs BP, $\mathrm{F}=66.91, P$-values $<0.001$ ). BP patients showed the highest IQ while SCZ patients showed the lowest. After controlling for IQ and all demographic factors, both SCZ patients and BP patients showed significantly higher error rates on the two working memory tasks than did the controls (all $P$-values $<0.001$ ). The differences between the two patient populations were not significant for both tasks (all $P$-values $>0.05$ ).

No deviation from Hardy-Weinberg equilibrium was found in controls, patients, or the total sample (all $P$-values $>0.05$ ). There were no significant differences in genotype frequencies between SCZ patients and controls and between BP patients and controls (all $P$-values $>0.05$ ). Moreover, there were no significant differences in all demographic factors across genotypes in all three samples (all $P$-values $>0.05$, see Table 2).

Significant interactions between genotype and diagnosis were found for the error rates of the 1-back task $(\mathrm{F}=10.281$, $P<0.001)$ and the BX condition of the DPX task $(\mathrm{F}=6.083$, $P=0.002)$. Moreover, there was a marginally significant interaction for the error rate of the 2-back task $(\mathrm{F}=2.640$, $P=0.072$ ). For BX-AY difference scores, the main effect of genotype was significant $(\mathrm{F}=4.265, P=0.039)$, but the interaction between genotype and diagnosis was not significant $(\mathrm{F}=0.811, P=0.445)$ (Table 3$)$. To explore the nature of the interactions between genotype and diagnosis as well as the main effect of genotype across samples, we 
Table 2 Differences in the Demographic Data Across CACNA / C rs 1006737 Genotype for Patients and Healthy Controls

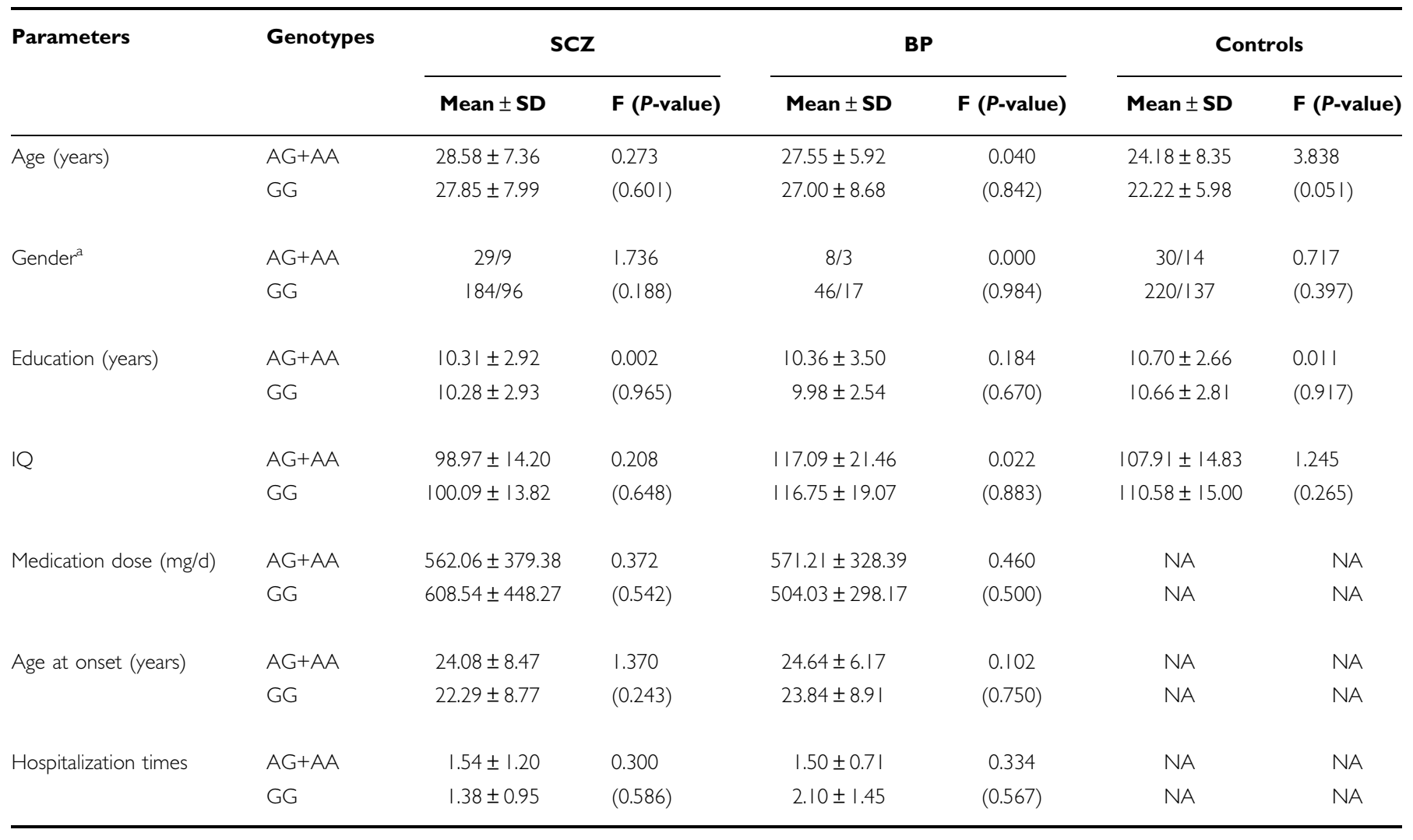

Abbreviation: NA, not applicable.

${ }^{a} \chi^{2}$ test.

Table 3 Cognitive Functions According to CACNA / C rs 1006737

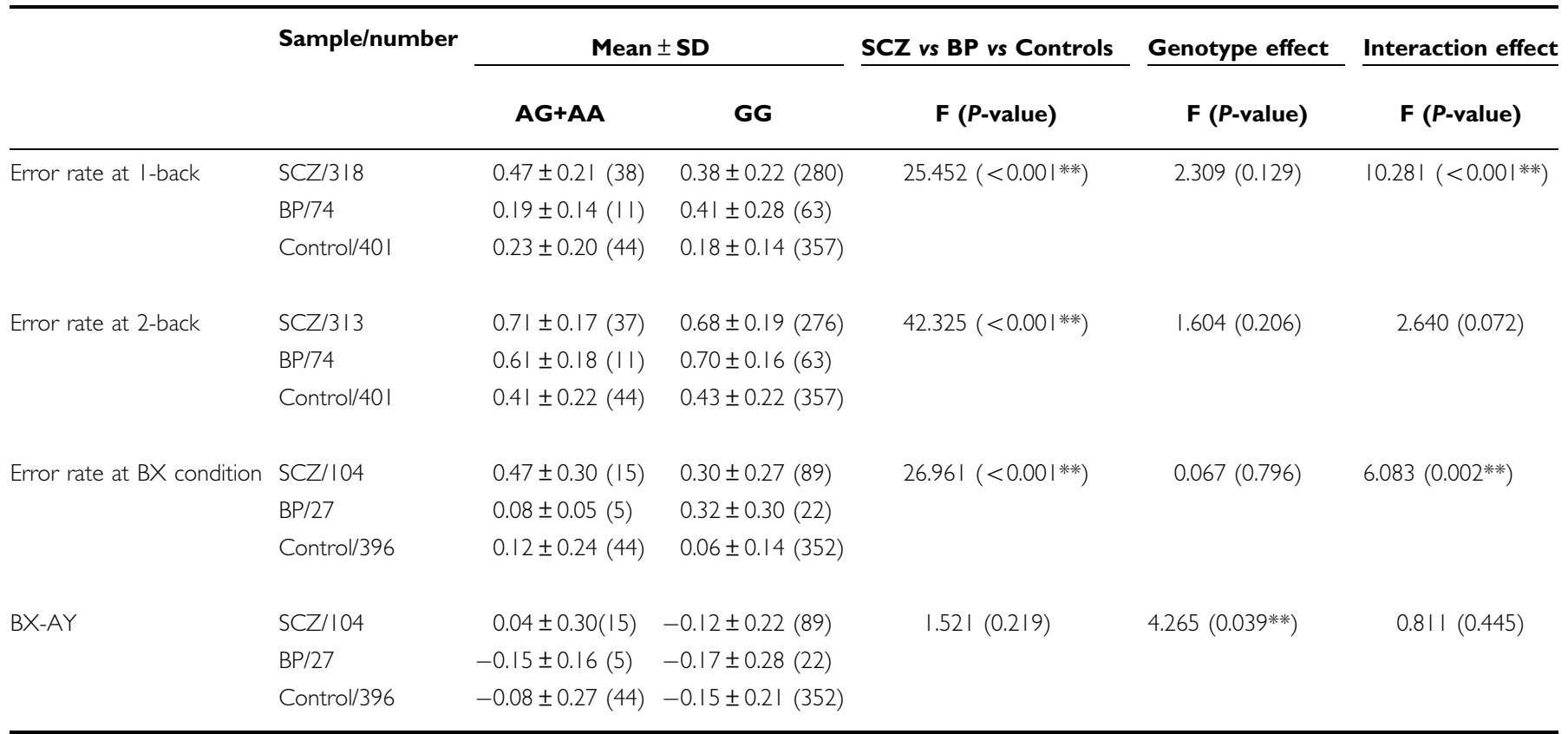

*** $P$-value $<0.05$.

then examined the simple effects of genotype in the three samples separately. For the 1-back task, significant associations between genotype and working memory were found in all three samples. The direction of association was the same in SCZ patients $(\mathrm{F}=4.764, P=0.030)$ and controls $(\mathrm{F}=6.046, P=0.014$ ) with the A allele (the clinical risk allele) carriers showing significantly higher error rates than did the G allele homozygotes. However, BP patients showed 
an opposite direction of association, with $\mathrm{A}$ allele carriers making fewer errors than $\mathrm{G}$ allele homozygotes $(\mathrm{F}=8.152$, $P=0.006)$. Analysis on the error rates of the $\mathrm{BX}$ condition showed similar results. Compared with the GG genotype, the A allele carriers made more errors if they were either SCZ patients $(\mathrm{F}=4.005, P=0.048)$ or controls $(\mathrm{F}=4.643, P=0.032)$, but made fewer errors if they were BP patients $(\mathrm{F}=3.180, P=0.087)$. For BX-AY difference scores, A allele carriers were significantly higher than $G$ allele homozygotes in both SCZ patients $(\mathrm{F}=5.562$, $P=0.020)$ and controls $(\mathrm{F}=4.297, P=0.039)$, but no significant genotype effect was found in BP patients $(\mathrm{F}=0.029, P=0.867)$ (see Figure 1$)$.

Finally, to investigate whether the genetic effects were of the same magnitude for SCZ patients and the controls, we did a two-way ANOVA. Results showed no interaction effects (for the 1-back task, $\mathrm{F}=0.488, P=0.485$; for the 2-back task, $\mathrm{F}=1.205, P=0.273$; and for the DPX task, $\mathrm{F}=3.167, \quad P=0.076), \quad$ suggesting consistent genetic effects for these two groups. The main effects of genotype were significant for the 1-back task $(\mathrm{F}=9.889, P=0.002)$, and the BX condition of the DPX task $(\mathrm{F}=13.630$, $P<0.001)$, and the BX-AY difference scores $(\mathrm{F}=10.134$, $P=0.002)$.

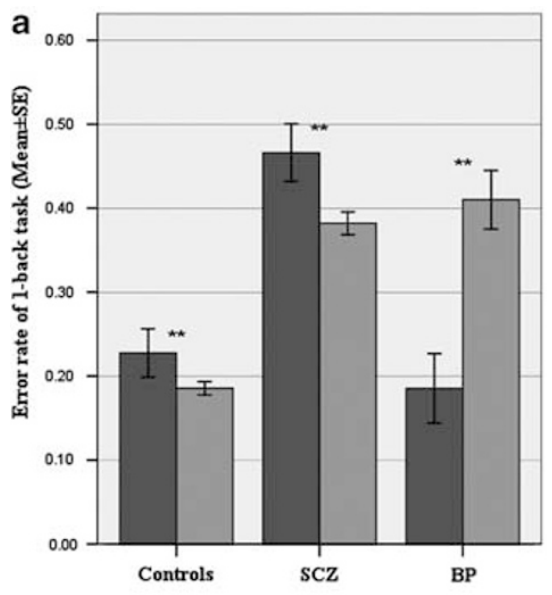

\section{DISCUSSION}

The current study was the first to report significant associations between rs1006737 and behavioral measures of working memory. Consistent with our hypothesis, both SCZ patients and healthy controls with the clinical risk allele showed significantly higher error rates on the 1-back task and the BX condition of the DPX task as well as higher BXAY error difference scores than did their major allele homozygote counterparts. In other words, the clinical risk allele was associated with impaired working memory. In contrast, patients with BP showed a counterintuitive, reversed direction of associations for the 1-back task and the BX condition of the DPX task. These results suggest that rs1006737 has an important role in working memory, but its mechanisms may vary by patient groups.

First, for healthy controls, the current results were consistent with previous studies. Using the fMRI method, Bigos et al (2010) and Krug et al (2010) found that the risk allele was associated with increased activity of the DLPFC - an indication of poorer working memory. Studying mostly the same subjects as those of Krug et al's (2010) study, Thimm et al (2011) found that, compared with other genotypes, the rs1006737 risk allele homozygotes showed
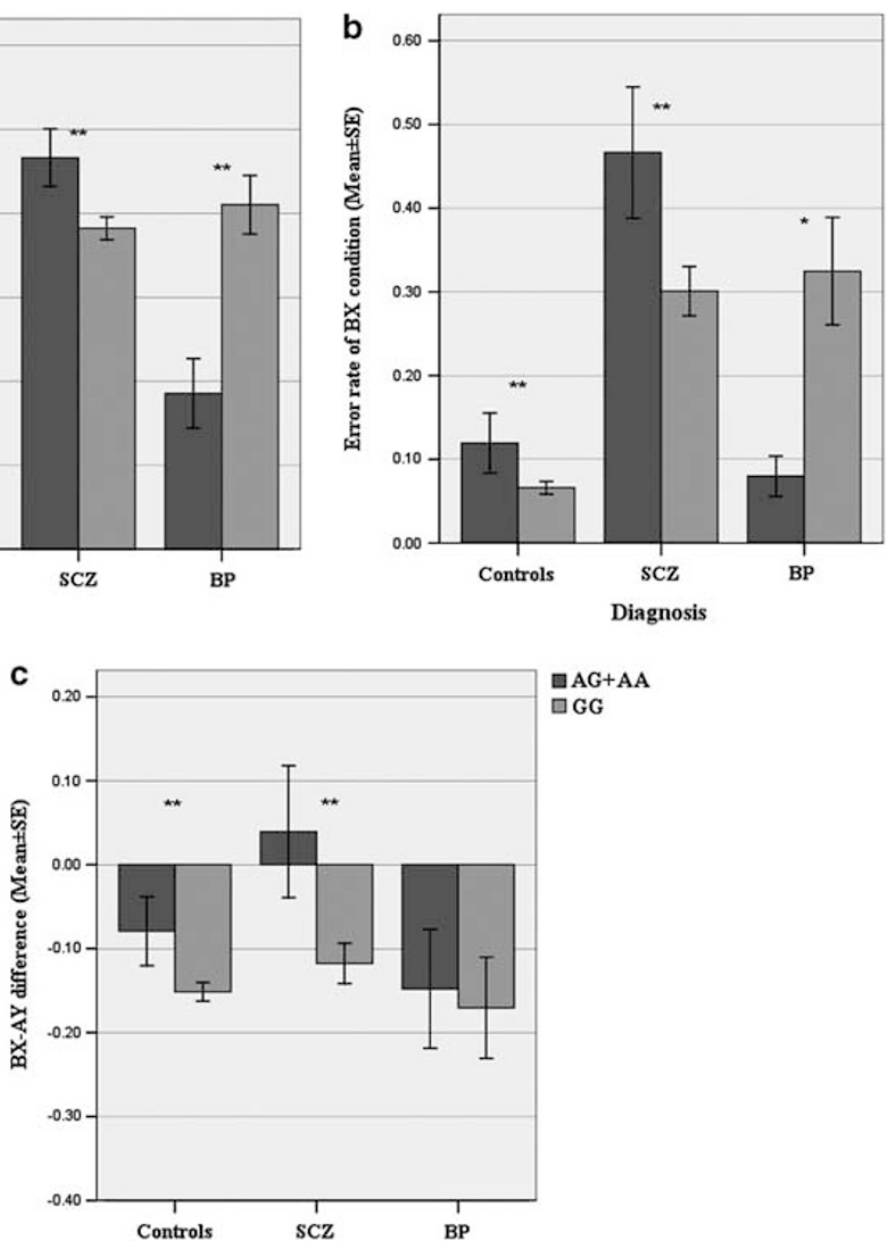

Figure I Working memory by rs 1006737 genotype and diagnosis. For SCZ patients and healthy controls, compared with the major allele (G) homozygotes, clinical risk allele (A) carriers made more errors at both the I-back task (the panel a) and the BX condition of the DPX task (the panel b) and showed higher BX-AY difference scores (the panel c). In contrast, for BP patients, A carriers made fewer errors at the I-back task and the BX condition than G homozygotes. $* P<0.1$, $* * P<0.05$. 
slightly higher (though not statistically significant) conflict effect at the behavioral level and significantly reduced neural activity in the frontal gyrus, again suggesting a link between the risk allele and impaired cognitive function.

As for SCZ and BP, several lines of previous research are relevant to the explanation of our finding of disease-specific associations between rs 1006737 and working memory. First, although patients with BP and SCZ showed similar deficits of working memory in this study as in previous studies (Hill et al, 2008; McGrath et al, 2001), it does not necessarily mean that both diseases have the same neural basis for their impaired working memory. A recent meta-analysis of studies on SCZ patients revealed decreased activity in the DLPFC, reflecting disrupted frontal-based top-down control functions, but increased activity in the VLPFC, suggesting a compensatory response (Minzenberg et al, 2009). In contrast, another meta-analysis of research on BP patients found that these patients, especially those with mania episodes, show decreased activity in the VLPFC, but no changes in the DLPFC (Chen et al, 2011). The differences in neural bases of impaired working memory between SCZ and $\mathrm{BP}$ patients have also been confirmed by several studies that directly compared these two groups. It should be noted that these studies used different techniques and measures. For example, some used the fMRI technique to measure prefrontal activity related to working memory (Curtis et al, 2001; Costafreda et al, 2011; McIntosh et al, 2008), others used the structural MRI technique to measure the gray matter volumes of the prefrontal cortex (Molina et al, 2011), and still others used the magnetic resonance spectroscopy technique to measure the NAA concentration (Molina et al, 2007) in the prefrontal cortex.

Second, our particular finding that the clinical allele was associated with better working memory in patients with BP is similar to a finding involving rs1344706 of the ZNF804 gene (Walters et al, 2010). This SNP is the sole polymorphism that could withstand whole-genome corrections in whole-genome scan studies of SCZ (O'Donovan et al, 2008). Walters et al (2010) recently found that the clinical risk allele was also associated with better cognitive functions. These authors further tested the association between SCZ and this SNP in a subgroup of patients with relatively intact cognitive functions and found that the association became stronger with increasing cognitive function, suggesting interactive effects among cognitive functions, disease, and genes.

Third, the only previous study involving rs 1006737 and BP patients (Perrier et al, 2011) found opposite directions of association in BP and healthy controls for the gray matter density of the left putamen. Specifically, the clinical risk allele was associated with decreased gray matter density in BP patients but increased gray matter density in healthy controls. The putamen is a substructure of the basal ganglia and has projections to the prefrontal cortex, thus is also involved in the etiology of working memory (Chang et al, 2004). A recent meta-analysis indicated that BP patients actually had enlarged putamen volume (Hallahan et al, 2011). In other words, just like our results, Perrier and colleagues' results on BP patients were also counterintuitive. Taken together, it is likely that the role of rs1006737 in BP patients' brain functions may differ from those in healthy controls or patients with other diseases. Future research may also examine the disease-specific effects of rs1006737 in brain regions beyond the prefrontal cortex, because several structural and functional MRI studies have found that healthy subjects with the clinical risk allele showed altered volumes or activities in brain regions such as the hippocampus (Bigos et al, 2010; Erk et al, 2010), amygdala (Wessa et al, 2010), and other regions (Kempton et al, 2009; Franke et al, 2010).

Although our results were consistent for the 1-back and DPX tasks, we did not find significant genetic effects (except for one marginal effect) on the 2-back task. This result may have been due to the complex nature of the 2-back task, which is more difficult (ie, higher error rates) than the other two tasks and likely to involve different cognitive processes. For example, fMRI studies on the N-back tasks (Callicott et al, 1999; Van Snellenberg et al, 2006; Manoach, 2003) have revealed that, at lower working memory load for the 1-back task, DLPFC activity increases monotonically with the increasing load. However, the relationship between DLPFC activity and working memory load becomes nonmonotonic or inverted U-shaped when working memory load increases beyond a subject's ability to effectively perform the task (Callicott et al, 1999; Van Snellenberg et al, 2006; Manoach, 2003). It is likely that the 2-back task involves more than the DLPFC, especially among the lowperforming subjects who showed poor effort, frustration, or guessing (Van Snellenberg et al, 2006). If that is the case, the simpler 1-back task may be more sensitive to the DLPFC function and related working memory than the 2-back task. The former may also be a more applicable endophenotype of these disorders than the latter.

The results of our study are intriguing, but replications are needed before we can draw conclusions confidently about disease-specific genetic effects on working memory. First, the sample size of patients with BP was quite small, especially for the DPX task. It is worth noting that previous studies have published results with similarly small sample sizes when using quantitative cognitive performance as a phenotype. For an instance, Opgen-Rhein et al (2008) published a study in Schizophrenia Bulletin which contained $63 \mathrm{SCZ}$ patients (involving $11 \mathrm{val} / \mathrm{val}$ ) and 40 healthy controls (involving $13 \mathrm{val} / \mathrm{val}$ and $6 \mathrm{met} / \mathrm{met}$ ) and found significant associations between COMT gene polymorphism and ANT task performance. In this study, we obtained consistent results across two different measures of spatial working memory, but it is necessary to replicate these results with large samples.

Second, our failure to find significant differences of genotype frequencies between patients and controls might also have been due to sample size limitations. The current opinion is that a large sample size of a thousand is needed to discover common polymorphisms for common diseases. For example, in the previous case-control studies of rs1006737, the smallest sample sizes were 479 patients and 11361 controls in studies of SCZ (Green et al, 2010) and 1098 patients and 1267 controls in studies of BP (Ferreira et al, 2008). Although our limited sample size may have been responsible for the lack of significant association between genotype and diagnosis, it was large enough to examine the associations between genotype and the quantitative endophenotype (ie, working memory) of the BP and SCZ diseases (even for the small sample of BP patients). 
Finally, some demographic factors, especially IQ and age, were not well matched across the three samples. Although these demographic factors were included as covariates in all the analyses on the associations between genotype and working memory, better-matched samples should yield more convincing results. In addition, with patients as subjects, potential confounds such as the effect of medications and patients' impaired cognitive functions also need to be considered in future research.

In conclusion, the current study found significant associations between rs1006737 and working memory in three samples, including healthy controls, patients with SCZ, and patients with BP. The direction of association was the same for SCZ patients and healthy controls, but reversed for BP patients, suggesting possible disease-specific genetic effects on working memory. This finding adds to the emerging literature on complex interactions among genes, diseases, and brain functions and pointed to the necessity for more studies in patient populations.

\section{ACKNOWLEDGEMENTS}

This work was supported by the Natural Science Foundation of China (30800294), the Medical Science and Technology Plan of Shandong Province (2009QZ003), and the Fundamental Research Funds for the Central Universities.

\section{DISCLOSURE}

The authors declare no conflict of interest.

\section{REFERENCES}

Barch DM, Berman MG, Engle R, Jones JH, Jonides J, Macdonald A et al (2009). CNTRICS final task selection: working memory. Schizophr Bull 35: 136-152.

Barch DM, Carter CS, Arnsten A, Buchanan RW, Cohen JD, Geyer $\mathrm{M}$ et al (2009). Selecting paradigms from cognitive neuroscience for translation into use in clinical trials: proceedings of the third CNTRICS meeting. Schizophr Bull 35: 109-114.

Bigos KL, Mattay VS, Callicott JH, Straub RE, Vakkalanka R, Kolachana B et al (2010). Genetic variation in CACNA1C affects brain circuitries related to mental illness. Arch Gen Psychiatry 67: 939-945.

Blokland GA, McMahon KL, Hoffman J, Zhu G, Meredith M, Martin NG et al (2008). Quantifying the heritability of taskrelated brain activation and performance during the N-back working memory task: a twin fMRI study. Biol Psychol 79: 70-79.

Callicott JH, Mattay VS, Bertolino A, Finn K, Coppola R, Frank JA et al (1999). Physiological characteristics of capacity constraints in working memory as revealed by functional MRI. Cereb Cortex 9: $20-26$.

Callicott JH, Ramsey NF, Tallent K, Bertolino A, Knable MB, Coppola $\mathrm{R}$ et al (1998). Functional magnetic resonance imaging brain mapping in psychiatry: methodological issues illustrated in a study of working memory in schizophrenia. Neuropsychopharmacology 18: 186-196.

Cardno AG, Rijsdijk FV, Sham PC, Murray RM, McGuffin P (2002). A twin study of genetic relationships between psychotic symptoms. Am J Psychiatry 159: 539-545.

Catterall WA (2000). Structure and regulation of voltage-gated Ca2+ channels. Annu Rev Cell Dev Biol 16: 521-555.

Chang K, Adleman NE, Dienes K, Simeonova DI, Menon V, Reiss A et al (2004). Anomalous prefrontal-subcortical activation in familial pediatric bipolar disorder: a functional magnetic resonance imaging investigation. Arch Gen Psychiatry 61: 781-792.

Chen CH, Suckling J, Lennox BR, Ooi C, Bullmore ET (2011). A quantitative meta-analysis of fMRI studies in bipolar disorder. Bipolar Disord 13: 1-15.

Costafreda SG, Fu CH, Picchioni M, Toulopoulou T, McDonald C, Kravariti E et al (2011). Pattern of neural responses to verbal fluency shows diagnostic specificity for schizophrenia and bipolar disorder. BMC Psychiatry 11: 18.

Curtis VA, Dixon TA, Morris RG, Bullmore ET, Brammer MJ, Williams SC et al (2001). Differential frontal activation in schizophrenia and bipolar illness during verbal fluency. J Affect Disord 66: 111-121.

Erk S, Meyer-Lindenberg A, Schnell K, Opitz von Boberfeld C, Esslinger C, Kirsch P et al (2010). Brain function in carriers of a genome-wide supported bipolar disorder variant. Arch Gen Psychiatry 67: 803-811.

Ferreira MA, O'Donovan MC, Meng YA, Jones IR, Ruderfer DM, Jones L et al (2008). Collaborative genome-wide association analysis supports a role for ANK3 and CACNA1C in bipolar disorder. Nat Genet 40: 1056-1058.

Franke B, Vasquez AA, Veltman JA, Brunner HG, Rijpkema M, Fernández G (2010). Genetic variation in CACNA1C, a gene associated with bipolar disorder, influences brainstem rather than gray matter volume in healthy individuals. Biol Psychiatry 68: $586-568$.

Green EK, Grozeva D, Jones I, Jones L, Kirov G, Caesar S et al (2010). The bipolar disorder risk allele at CACNA1C also confers risk of recurrent major depression and of schizophrenia. Mol Psychiatry 15: 1016-1022.

Hallahan B, Newell J, Soares JC, Brambilla P, Strakowski SM, Fleck $\mathrm{DE}$ et al (2011). Structural magnetic resonance imaging in bipolar disorder: an international collaborative mega-analysis of individual adult patient data. Biol Psychiatry 69: 326-335.

Hill SK, Harris MS, Herbener ES, Pavuluri M, Sweeney JA (2008). Neurocognitive allied phenotypes for schizophrenia and bipolar disorder. Schizophr Bull 34: 743-759.

Kempton MJ, Ruberto G, Vassos E, Tatarelli R, Girardi P, Collier D et al (2009). Effects of the CACNA1C risk allele for bipolar disorder on cerebral gray matter volume in healthy individuals. Am J Psychiatry 166: 1413-1414.

Krug A, Nieratschker V, Markov V, Krach S, Jansen A, Zerres K et al (2010). Effect of CACNA1C rs1006737 on neural correlates of verbal fluency in healthy individuals. Neuroimage 49: 1831-1836.

MacDonald AW, Goghari VM, Hicks BM, Flory JD, Carter CS, Manuck SB (2005). A convergent-divergent approach to context processing, general intellectual functioning, and the genetic liability to schizophrenia. Neuropsychology 19: 814-821.

MacDonald AW, Pogue-Geile MF, Johnson MK, Carter CS (2003). A specific deficit in context processing in the unaffected siblings of patients with schizophrenia. Arch Gen Psychiatry 60: 57-65.

Manoach DS (2003). Prefrontal cortex dysfunction during working memory performance in schizophrenia: reconciling discrepant findings. Schizophr Res 60: 285-298.

McGrath J, Chapple B, Wright M (2001). Working memory in schizophrenia and mania: correlation with symptoms during the acute and subacute phases. Acta Psychiatr Scand 103: 181-188.

McIntosh AM, Whalley HC, McKirdy J, Hall J, Sussmann JE, Shankar P et al (2008). Prefrontal function and activation in bipolar disorder and schizophrenia. Am J Psychiatry 165: 378-384.

Minzenberg MJ, Laird AR, Thelen S, Carter CS, Glahn DC (2009). Meta-analysis of 41 functional neuroimaging studies of executive function in schizophrenia. Arch Gen Psychiatry 66: 811-822.

Molina V, Galindo G, Cortés B, de Herrera AG, Ledo A, Sanz J et al (2011). Different gray matter patterns in chronic schizophrenia 
and chronic bipolar disorder patients identified using voxel-based morphometry. Eur Arch Psychiatry Clin Neurosci 261: 313-322.

Molina V, Sánchez J, Sanz J, Reig S, Benito C, Leal I et al (2007). Dorsolateral prefrontal $\mathrm{N}$-acetyl-aspartate concentration in male patients with chronic schizophrenia and with chronic bipolar disorder. Eur Psychiatry 22: 505-512.

Moskvina V, Craddock N, Holmans P, Nikolov I, Pahwa JS, Green $\mathrm{E}$ et al (2009). Gene-wide analyses of genome-wide association data sets: evidence for multiple common risk alleles for schizophrenia and bipolar disorder and for overlap in genetic risk. Mol Psychiatry 14: 252-260.

Nyegaard M, Demontis D, Foldager L, Hedemand A, Flint TJ, Sorensen KM et al (2010). CACNA1C (rs1006737) is associated with schizophrenia. Mol Psychiatry 15: 119-121.

Obermair GJ, Tuluc P, Flucher BE (2008). Auxiliary $\mathrm{Ca}(2+)$ channel subunits: lessons learned from muscle. Curr Opin Pharmacol 8: 311-318.

O'Donovan MC, Craddock N, Norton N, Williams H, Peirce T et al (2008). Identification of loci associated with schizophrenia by genome-wide association and follow-up. Nat Genet 40: 1053-1055. Opgen-Rhein C, Neuhaus AH, Urbanek C, Hahn E, Sander T, Dettling M (2008). Executive attention in schizophrenic males and the impact of COMT Val108/158Met genotype on performance on the attention network test. Schizophr Bull 34: 1231-1239.

Perrier E, Pompei F, Ruberto G, Vassos E, Collier D, Frangou S (2011). Initial evidence for the role of CACNA1C on subcortical brain morphology in patients with bipolar disorder. Eur Psychiatry 26: 135-137.

Purcell S, Neale B, Todd-Brown K, Thomas L, Ferreira MA, Bender D et al (2007). PLINK: a tool set for whole-genome association and population-based linkage analyses. Am J Hum Genet 81: 559-575.

Si TM, Shu L, Dang WM, Su YA, Chen JX, Dong WT et al (2009). Evaluation of the reliability and validity of Chinese version of the MINI. International neuropsychiatric interview in patients with mental disorders. Chin Mental Health J 23: 493-497 (in China). Sklar P, Smoller JW, Fan J, Ferreira MA, Perlis RH, Chambert K et al (2008). Whole-genome association study of bipolar disorder. Mol Psychiatry 13: 558-569.

Thimm M, Kircher T, Kellermann T, Markov V, Krach S, Jansen A et al (2011). Effects of a CACNA1C genotype on attention networks in healthy individuals. Psychol Med 41: 1551-1561.

Van Snellenberg JX, Torres IJ, Thornton AE (2006). Functional neuroimaging of working memory in schizophrenia: task performance as a moderating variable. Neuropsychology 20: 497-510.

Walters JT, Corvin A, Owen MJ, Williams H, Dragovic M, Quinn EM et al (2010). Psychosis susceptibility gene ZNF804A and cognitive performance in schizophrenia. Arch Gen Psychiatry 67: 692-700.

Wessa M, Linke J, Witt SH, Nieratschker V, Esslinger C, Kirsch P et al (2010). The CACNA1C risk variant for bipolar disorder influences limbic activity. Mol Psychiatry 15: 1126-1127. 